\title{
Crescimento físico em estatura de escolares de ascendência japonesa na cidade de São Paulo, Brasil
}

\author{
Physical growth in stature of school children of \\ J apanese ancestry in the city of São Paulo, Brazil
}

Gil berto Kac 1

Ricardo V. Santos 1, 2

\footnotetext{
1 Departamento de Endemias Samuel Pessoa, Escola Nacional de Saúde Pública, Fundação Oswaldo Cruz. Rua Leopoldo Bul hões 1480, Rio de Janeiro, RJ, 21041-210, Brasil.

2 Departamento de Antropologia, Museu Nacional, Universidade Federal do Rio de Janeiro. Quinta da Boa Vista s/no, Rio de Janeiro, RJ, 20940-040, Brasil.
}

Abstract This paper reports the results of a cross-sectional survey describing the growth in stature of a sample of school children of Japanese ancestry (three or four grandparents born in Japan) and of high soci o-economic status living in São Paulo, Brazil. The sample comprises 124 individuals 7-10 years of age, of both sexes. The results show that the children present mean values of stature bel ow the median values of the NCHS curves ("National Center for Heal th Statistics", U.S.A.). The values are similar to the medians of a sample of Japanese children and bel ow those of well-to-do Brazilian children of non-Japanese ancestry studied by the PNSN ("Pesquisa Nacional sobre Saúde e Nutrição", Brazil). The findings do not support the hypothesis of uniformity of growth potential in stature, casting doubts on the utilization of a single anthropometric reference in the assessment of nutritional status.

Key words Physical Growth; Stature; Japanese-Brazilians; Anthropometry; Nutrition

Resumo Este trabalho apresenta os resultados de um inquérito transversal que visou caracterizar o crescimento físico em estatura de uma amostra de escolares deascendência japonesa (três ou quatro avós nasci dos no Japão) e de al to status sóci o-econômi co resi dentes na ci dade de São Paulo. A amostra inclui 124 indi víduos entre 7 e 10 anos de idade e de ambos os sexos. Os resultados demonstram que as crianças de ascendência japonesa apresentam médias de estatura inferiores às medianas das curvas da referência NCHS ("National Center for Health Statistics", E.U.A.). As médias são si mi lares às medianas de uma amostra de crianças japonesas e inferiores àquelas de maior renda da PNSN ("Pesquisa Nacional sobre Saúdee Nutrição", Brasil ). Os achados não apóiam a hipótese de uniformidade do potencial de crescimento físi co em estatura em crianças, Iançando dúvidas acerca da utilização de uma única referência de cresci mento na avaliação antropométrica do estado nutricional.

Palavras-chave Crescimento Físico; Estatura; Nipo-brasilei ros; Antropometria; Nutrição 
Introdução

O acompanhamento do crescimento físico através da antropometria é parte essencial de aval iações das condições de saúde e nutrição de crianças em idade escolar. A Organização Mundial da Saúde (OMS) preconiza a utilização das curvas de crescimento de crianças norte-americanas na avaliação antropométrica do estado nutricional, quais sejam, aquelas compiladas pelo "National Center for Health Statistics" (NCHS) (Waterlow et al., 1977; WHO, 1986, 1995). Esta recomendação parte da premissa da inexistência de diferenças no potencial genético relativo ao crescimento físico em crianças bem nutridas de diferentes etnias, 0 que se constitui na proposta de "uma única referência para todos" (Habicht et al., 1974; Graitcer \& Gentry, 1981). É importante ressaltar, contudo, que não há unanimidade com relação a esta proposição (Jelliffe \&Jelliffe, 1989; Eveleth $\&$ Tanner, 1990). Estudos enfocando grupos populacionais específicos, como crianças de origem asiática, têm questionado a validade do emprego das curvas do NCHS como padrão em avaliações antropométricas do estado nutricional, notadamente com relação ao parâmetro estatura (Van Loon et al., 1986; Davies, 1988).

Este trabal ho reporta dados de um inquérito antropométrico realizado em uma amostra de escolares brasileiros de ascendência japonesa e de alto status sócio-econômico residentes na cidade de São Paulo. Os dados são discutidos à luz da hipótese da uniformidade do potencial genético relativo ao crescimento físico em estatura.

Material e métodos

Foram coletados dados de estatura de 271 escolares de 7 a 10 anos, estudantes de três escolas privadas e de alto status sócio-econômico da cidade de São Paulo, freqüentadas principalmente por crianças de ascendência japonesa nascidas no Brasil. Para fins deste trabalho, optou-se por apresentar somente os dados dos escolares com três ou quatro avós nascidos no Japão ( $n=124$ ) (Tabela 1). A estatura foi aferida por dois observadores previamente treinados, seguindo Lohman et al. (1988) e utilizando antropômetro (GPM) com precisão de $0,1 \mathrm{~cm}$. Permissão para participação no estudo, data de nascimento e local de nascimento dos avós das crianças foram obtidos através de questionários respondidos pelos pais dos alunos. As idades foram calculadas a partir das datas de nascimento, seguindo recomendação de Eveleth \& Tanner (1990:8).

A análise antropométrica baseou-se no indicador estatura para idade (E/I). Valores de escores-Z e de percentis foram calculados a partir de comparação com população de referência norte-americana compilada pelo NCHS ("National Center for Health Statistics") (Hamill et al., 1979).

Os pacotes EPI- 5 e SPSS/ $\mathrm{PC}+5.0$ foram empregados na análise quantitativa dos dados antropométricos.

\section{Resultados}

Do total de 124 crianças estudadas, apenas $4,0 \%$ ( $6,1 \%$ dos meninos e $1,7 \%$ das meninas) apresentaram valores de estatura para idade inferiores a -2 desvios-padrão em relação às medianas do NCHS. Ao nível de 5\%, não foi detectada diferença estatisticamente significante $(t=+1,51,122$ g.l., $p=0,134)$ entre os sexos nos valores médios de escore- $Z$ ( $x=-0,271$, D.P. $=$ 1,041 para meninos e $x=-0,529, D . P .=0,837$ para meninas). Tampouco foi observada diferença entre as médias do escore- $Z$ ao se comparar crianças com três e quatro avós japoneses ( $\mathrm{t}=$ $-0,20,122$ g.l., $p=0,843$ ).

Tabela 1

Distribuição da amostra por sexo, idade e número de avós japoneses, São Paulo, 1994.

\begin{tabular}{|c|c|c|c|c|c|c|c|}
\hline \multirow[t]{2}{*}{ Idade } & \multicolumn{3}{|c|}{ Meninos } & \multicolumn{3}{|c|}{ Meninas } & \multirow{2}{*}{$\begin{array}{c}\text { Sexos combinados } \\
\text { total geral }\end{array}$} \\
\hline & 3 avós & 4 avós & sub-total & 3 avós & 4 avós & sub-total & \\
\hline 7 anos & 04 & 09 & 13 & 04 & 01 & 05 & 18 \\
\hline 8 anos & 07 & 05 & 12 & 04 & 11 & 15 & 27 \\
\hline 9 anos & 13 & 11 & 24 & 06 & 13 & 19 & 43 \\
\hline 10 anos & 04 & 13 & 17 & 08 & 11 & 19 & 36 \\
\hline Total & 28 & 38 & 66 & 22 & 36 & 58 & 124 \\
\hline
\end{tabular}


A Figura 1 apresenta a distribuição decilar do indicador estatura/ idade para os sexos combinados, que demonstra uma concentração à esquerda, com $31 \%$ e $46 \%$ da amostra situando-se nos dois e três primeiros decis, respectivamente. Tal observação também se aplica para os sexos em separado. Assim, 30,3\% da amostra de meninos e $32,7 \%$ da de meninas encontram-se nos dois primeiros decis, quando o esperado seria $20 \%$.

A Figura 2 compara os valores médios de escore-Z segundo idade, computados em relação à mediana do NCHS, com dados oriundos de um estudo nacional conduzido no Japão (Eveleth \& Tanner, 1990:284-86) e com dados da "Pesquisa Nacional sobre Saúde e Nutrição" (PNSN), realizada em 1989 (INAN, 1990). Neste caso são apresentadas as estaturas medianas das crianças do primeiro e quarto quartis de renda. Para os escolares paulistas de ascendência japonesa, as médias são consistentemente negativas e se sobrepõem aos valores da amostra japonesa, com exceção da faixa etária dos 7 anos. Os dados das meninas também apontam para valores consistentemente negativos e mais próximos, ainda que não necessariamente sobrepostos, aos da amostra japonesa. Além disso, a estatura das crianças paulistas de ascendência japonesa é consistentemente superior àquela da amostra de crianças brasileiras de baixa renda e bastante inferior àquela das crianças de maior renda medidas na PNSN.

\section{Discussão}

Os resultados deste estudo corroboram achados de outras investigações conduzidas com crianças de origem asiática tanto em seus países de origem (Van Loon et al., 1986; Davies, 1988; Eveleth \& Tanner, 1990) como em filhos de migrantes realizadas em outras partes do mundo, como nos Estados Unidos (Greulich, 1976), Inglaterra (Tanner et al., 1982) e Jamaica (Ashcroft et al., 1966), entre outros. Os resultados destes trabalhos indicam que crianças de origem asiática bem nutridas e de alto status sócio-econômico tendem a apresentar valores médios de estatura para idade inferiores àqueles das referências norte-americanas e européias selecionadas para efeito de comparação. Portanto, em consonância com estudos realizados em outros países, os dados de estatura para as crianças brasileiras de ascendência japonesa aqui reportados não apóiam a hipótese de uniformidade do potencial de crescimento físico em estatura. Estão, portanto, de acordo com Eveleth \& Tanner (1990:1), que afirmam
Figura 1

Distribuição decilar do indicador estatura/idade em escolares de ascendência japonesa da cidade de São Paulo de 7-10 anos, sexos combinados, 1994.

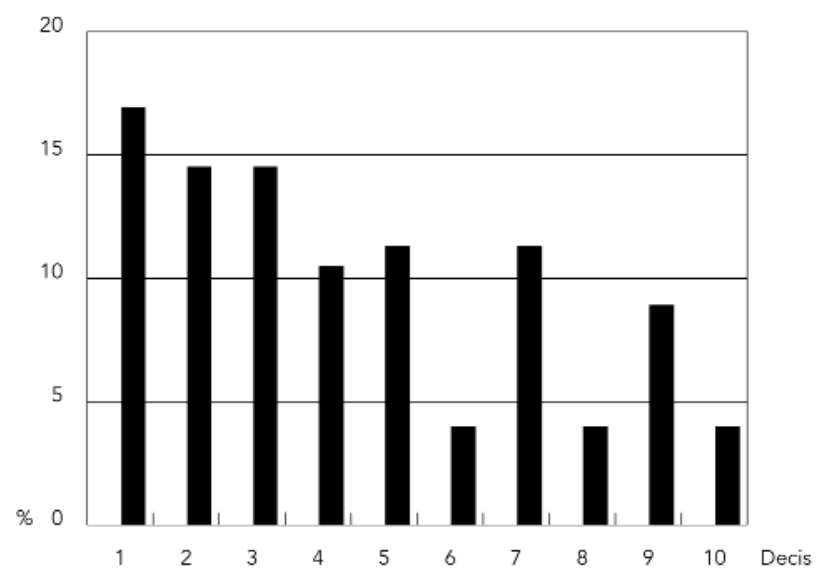

Figura 2

Médias de escore- $Z$ para o indicador estatura/idade em escolares de ascendência japonesa da cidade de São Paulo de 7-10 anos, segundo sexo, comparando-as com dados da PNSN ( 1 e e 4 quartis de renda) e com dados de crianças japonesas. Meninos

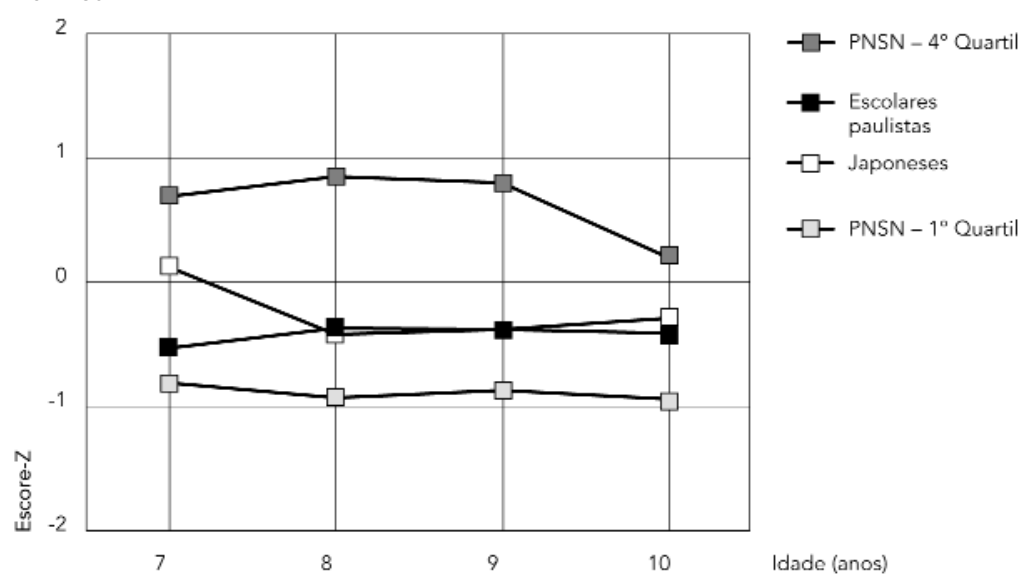

Meninas

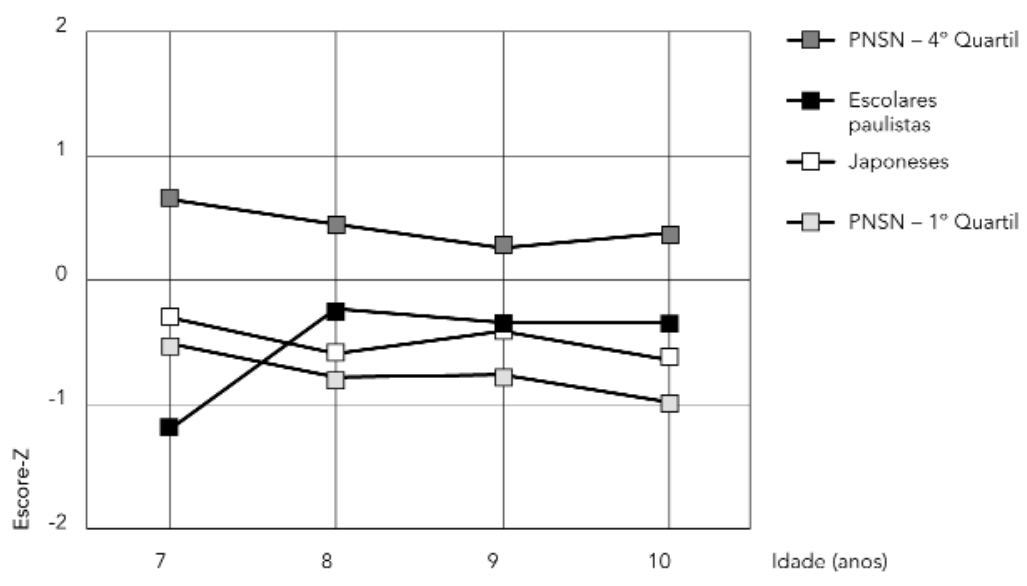


que "there is no guarantee [...] that all populations have the same growth potential. There are certainly large differences between populations [...] and it is now clear that a portion of these differences is genetic in origin".

É importante ressaltar que as crianças estudadas, apesar de valores médios de escore- $Z$ para estatura para idade inferiores às medianas da referência internacional, não apresentam elevadas prevalências de baixa estatura para idade (escore- $Z<-2$ ), que é da ordem de $4 \%$ somente. Ou seja, seguindo-se as recomendações da OMS (1986, 1995), a amostra estudada não se caracteriza por acentuada prevalência de desnutrição energético-protéica crônica. Deve-se mencionar, contudo, que no caso de estresse nutricional de longa duração, estas crianças de ascendência japonesa, por já apresentarem valores médios de escore- $Z$ consistentemente negativos e inferiores àqueles da referência, tenderão a cruzar de forma mais rápida o ponto de corte definidor do estado de desnutrição crônica (escore-Z<-2).

$\mathrm{O}$ que os dados aqui reportados potencialmente informam acerca da utilização de curvas internacionais de referência, como o NCHS, na avaliação do estado nutricional de crianças de ascendência japonesa nasci das no Brasil? Estudos sociológicos mostram que foi significativo o fluxo de migrantes japoneses para o Brasil nas primeiras décadas deste século e que, no presente, há regiões do país, como em diversas localidades no estado de São Paulo, com grandes concentrações de descendentes de japoneses (Handa, 1987; IBGE, 1982). Teoricamente, é plausível argumentar que nestes locais, e notadamente naqueles onde as condições sócio-econômicas e sanitárias estão aquém do adequado, inquéritos epidemiológicos poderão gerar uma estimativa inflacionada dos níveis de desnutrição energético-protéica, caso sejam empregados os pontos de corte recomendados pela OMS na avaliação do estado nutricional.

É escassa a literatura enfocando o crescimento físico de crianças de ascendência japonesa no Brasil (Guaraciaba \& Scalzo Fillho, 1963; Eveleth \& Tanner, 1990:180-3). Estudos mais detalhados, preferencialmente longitudinais e baseados em grandes amostras, precisam ser realizados visando verificar se, como sugerido neste trabalho e em outros da literatura internacional, as crianças de ascendência asiática bem-nutridas efetivamente apresentam, do ponto de vista populacional, perfil de crescimento em estatura distinto daquele das referências internacionais usualmente empregadas em avaliações do estado nutricional. Tais investigações revestem-se de importância face à proeminência de métodos antropométricos na interpretação do quadro nutricional em crianças e à possível peculiaridade do perfil de crescimento das crianças de ascendência asiática.

\section{Agradecimentos}

Os autores agradecem à Sílvia Gugelmin e a Rubens Ianelli pelo apoio durante o trabalho de coleta de dados em São Paulo e às Escolas Pioneiro, Nibra Park e Professor Oshimã pela permissão para realização do estudo. 


\section{Referências}

ASHCROFT, M. T.; HENEAGE, P. \& LOVELL, H. A., 1966. Heights and weights of Jamaican schoolchildren of various ethnic groups. American Journal of Physical Anthropology, 24:35-44.

DAVIES, D. P., 1988. The importance of genetic influences of growth in early chilhood with particular reference to children of Asiatic origin. In: Linear Growth Retardation in Less Developed Countries.(J. C. Waterlow, ed.), pp. 75-90. New York: Vevey Raven Press.

EVELETH, P. B \& TANNER, J. M., 1990. Worldwide Variation in Human Growth. Cambridge: Cambridge University Press.

GRAITCER, L. P. \& GENTRY, M. E., 1981. Measuring children: One reference for all. Lancet, 2:297-299.

GREULICH, W. W., 1976. Some secular changes in the growth of American-born and native Japanese children. American Journal of Physical Anthropology, 45:553-568.

GUARACIABA, M. A. \& SCALZO FILHO, C., 1963. O crescimento e desenvolvimento infantis na população nipo-brasileira de Bauru. Revista do Museu Paulista, 14:542-583.

HABICHT, J. P.; MARTORELL, R.; MALINA, R. M. \& KLEIN, R. E., 1974. Height and weight standards for pre-school children. How relevant are ethnic differences in growth potencial? Lancet, 1:611-615.

HAMILL, P. V. V.; DRIZD, A. T.; JOHNSON, L. C.; REED, B. R.; ROCHE, F. A. \& MOORE, M. W., 1979. Physical growth: National Center for Health Statistics percentiles. American Journal of Clinical Nutrition, 32:607-629.

HANDA, T., 1987. O Imigrante Japonês. São Paulo: T. A. Queiroz/Centro de Estudos Nipo-Brasileiros.

JELLIFFE, D. B. \& JELLIFFE, E. F., 1989. Community Nutritional Assessment: With Special Reference to Less Technicaly Devel oped Countries. Oxford: Oxford University Press.
IBGE (INSTITUTO BRASILEIRO DE GEOGRAFIA ESTATÍSTICA), 1982. Dados Gerais, Migração, Instrução, Fecundidade e Mortalidade. Estado de São Paulo. Vol 1., Tomo 4, no 20. Rio de Janeiro: IBGE.

INAN (INSTITUTO NACIONAL DE ALIMENTAÇÃO E NUTRIÇÃO), 1990. Pesquisa Nacional sobre Saúde e Nutrição. Perfil de Crescimento da População Brasileira de 0 a 25 Anos. Brasília: INAN.

LOHMAN, T. G.; ROCHE, A. F. \& MARTORELL, R., 1988. Anthropometric Standardization Reference Manual. Champaign, Illinois: Human Kinetics.

TANNER, J.M; HAYASHI, M.A.; PREECE, M.A. \& CAMERON, N., 1982. Increase in length of leg relative to trunk in Japanese children and adults from 1957 to 1977: Comparison with British and with Japanese Americans. Annals of Human Biology, 9:411-423.

VAN LOON, H.; SAVERYS, V.; VUYLSTCKE, J. P.; VLIETINCK, R. F. \& EECKELS, R., 1986. Locals versus universals growth standards: the effect of using NCHS as universal reference. Annals of Human Biology, 13:347-357.

WATERLOW, J. C.; BUZINA, R.; KELLER, W.; LANE, J. M.; NICHAM AN, N. Z. \&TANNER, J. M., 1977. The presentation and use of height and weight for comparing nutritional status of groups of children under the age of 10 years. Bulletin of the World Health Organization, 55:489-498.

WHO (WORLD HEALTH ORGANIZATION), 1986. Use and interpretation of anthropometric indicators of nutritional status. Bulletin of theWorld Health Organization, 64:929-41.

WHO (WORLD HEALTH ORGANIZATION), 1995. Physical Status: the Use and Interpretation of Anthropometry. WHO Technical Report Series, no 854. Genève: WHO. 